\title{
ESTUDO DA PARTIÇÃO DE ALBUMINA DE SORO BOVINO EM SISTEMAS AQUOSOS BIFÁSICOS
}

\author{
MICHELLE COSTA PIGNATA* \\ RENATA CRISTINA FERREIRA BONOMO** \\ CRISTIANE MARTINS VELOSO*** \\ OLGA REINERT RAMOS GANDOLFI*** \\ RAFAEL DA COSTA ILHEU FONTAN** \\ MELQUESEDECK SATURNINO CABRAL OLIVEIRA***** \\ PAULO BONOMO******
}

\begin{abstract}
Neste trabalho, estudou-se a aplicação da extração líquido-líquido (ELL) de albumina de soro bovino (BSA) por meio de sistemas aquosos bifásicos (SAB), compostos por polietilenoglicol (PEG), sulfato de sódio e água. Foram determinados dados de equilíbrio de fases para sistemas aquosos bifásicos PEG/sulfato de sódio em temperatura ambiente. Também foram avaliadas a influência dos fatores $\mathrm{pH}$, temperatura, concentração de PEG e concentração de sal sob a partição da BSA, aplicando-se, inicialmente, um delineamento fatorial fracionado. Os resultados obtidos foram submetidos à análise de variância, sendo verificada a influência do $\mathrm{pH}$, da temperatura e da concentração de $\mathrm{Na}_{2} \mathrm{SO}_{4}$ na partição da BSA. Para as variáveis significativas, foi empregado um delineamento composto central rotacional (DCCR). Os resultados obtidos no DCCR foram submetidos à análise de regressão e superfície de resposta, sendo determinadas as melhores condições para a otimização da partição da proteína. A extração da BSA pelo SAB utilizando PEG/sulfato de sódio mostrou-se eficaz, utilizandose de reagentes e etapas de processos bastante simples.
\end{abstract}

PALAVRAS-CHAVE: PARTIÇÃO DE PROTEÍNA, SAB, BSA, DADOS DE EQUILÍBRIO.

*Mestre em Engenharia e Ciências de Alimentos, Universidade Estadual do Sudoeste da Bahia (UESB), Itapetinga-BA. E-mail: chellecp23@hotmail.com.

**Doutor em Ciência de Alimentos, Departamento de Tecnologia Rural e Animal (DTRA), Universidade Estadual do Sudoeste da Bahia (UESB), Itapetinga-BA. E-mail: bonomorcf@yahoo.com.br; rafaelfontan@yahoo. com.br.

***Doutora em Química, Departamento de Ciências Naturais (DCN), Universidade Estadual do Sudoeste da Bahia (UESB), Vitória da Conquista-BA. E-mail: crismveloso@yahoo.com.br;

****Discente do Programa de Pós-Graduação em Engenharia e Ciências de Alimentos, Universidade Estadual do Sudoeste da Bahia (UESB), Itapetinga-BA. E-mail: olga.gandolfi@hotmail.com

${ }^{* * * * *}$ Mestre em Ciências Ambientais, Universidade Estadual do Sudoeste da Bahia (UESB), Itapetinga-BA. E-mail: melque_engenheiro@yahoo.com.br

******Doutor em Genética e Melhoramento, Departamento de Ciências Exatas e Naturais (DCEN), Universidade Estadual do Sudoeste da Bahia (UESB), Itapetinga-BA. E-mail: bonomopaulo@yahoo.com.br 


\section{INTRODUÇÃO}

A extração líquido-líquido (ELL) com solventes orgânicos é uma operação muito utilizada na indústria química como técnica de separação. A extração de biocompostos em sistemas de duas fases líquidas imiscíveis constituídas de uma fase aquosa e um solvente orgânico é utilizada há cerca de 60 anos, na purificação de antibióticos e ácidos orgânicos. Para proteínas e biomoléculas, no entanto, tais sistemas não são adequados devido à sensibilidade desses componentes à desnaturação promovida pelos solventes orgânicos. Alternativamente, proteínas e outros materiais de origem biológica podem ser purificados em sistemas bifásicos constituídos por duas fases aquosas imiscíveis. A purificação é resultado de uma partição diferenciada da molécula-alvo e impurezas entre as duas fases líquidas (PESSOA e KILIKIAN, 2005).

Sistemas aquosos bifásicos (SABs) têm sido amplamente utilizados para recuperação e purificação de biomoléculas, como: proteínas, enzimas e antibióticos. Os SABs são um processo de separação simples e oferecem ambiente não tóxico suave para biomoléculas sensíveis, o que favorece a estabilidade delas durante a separação, quando se comparam com sistemas tradicionais, compostos com solventes orgânicos (SILVA, 2008). Recentes melhorias da técnica, com o emprego de novos SABs compostos por polímero e sal, polímero e polímero, permitem o seu uso em nível industrial (AZEVEDO et al., 2007).

Os SABs são formados por uma fase rica em polímero e a outra fase rica em outro polímero ou sal (COLLEN et al., 2004). As duas fases líquidas formadas encontram-se em equilíbrio termodinâmico, ou seja, as propriedades termodinâmicas intensivas como densidade, índice de refração e composição não estão variando em uma dimensão temporal, ou, ainda, não há troca resultante de matéria entre as fases. Essas fases estão separadas por uma interface que é a região na qual as propriedades termodinâmicas intensivas de cada fase possuem valores diferentes, sempre tendendo para o valor daquela propriedade no seio da outra fase em equilíbrio (CARVALHO, 2004).

O sistema polímero-polímero mais comum é composto de dextrana e PEG, mas a dextrana é de custo elevado, o que inviabiliza o seu uso em larga escala. Os sistemas PEG + sal são atrativos devido ao baixo custo, à alta seletividade e por apresentarem valores de viscosidade mais baixos. São sistemas capazes de ser formados à temperatura ambiente. A fase superior é composta majoritariamente pelo PEG, e a fase inferior, pelo sal. A separação de fases ocorre de forma rápida devido à diferença de densidade entre elas, o que facilita o uso de sistemas PEG + sal em larga escala industrial. Por outro lado, apresentam algumas limitações como a desnaturação de biomoléculas em altas concentrações de sal (SILVA e MEIRELLES, 2000).

Um amplo conjunto de variáveis, tais como temperatura, tipo e concentração de sal, concentração e massa molar do polímero, influencia a separação de fases em SABs e, consequentemente, o processo extrativo de um determinado biocomponente. A base da partição em SAB é a distribuição seletiva de compostos entre as duas fases. Por isso, a predição e a interpretação da partição de biopartículas em sistemas aquosos bifásicos são uma difícil tarefa, embora a manipulação das propriedades do sistema, tornando predominante um determinado tipo de interação, venha a ser uma forma de controlar a partição (HATTI-KAUL, 2001). Dessa forma, torna-se necessário estudar diferentes SABs para ampliar e facilitar o uso deles na extração líquidolíquido. A partir da obtenção das curvas de equilíbrio, as regiões monofásicas e bifásicas dos sistemas podem ser determinadas e, consequentemente, as concentrações globais dos componentes do sistema que dão origem a estas.

Segundo Cavalcanti et al. (2008), com a utilização dos delineamentos estatísticos, investigações experimentais podem ser realizadas mais rápida e economicamente.

Assim, objetivou-se neste trabalho o estudo da partição da proteína BSA (albumina de soro bovino) em sistemas aquosos bifásicos, compostos por polietileno glicol e sulfato de sódio em função da temperatura, concentração de sal, concentração de PEG e do pH utilizando-se os delineamentos fatorial fracionado $\left(2^{4-1}\right)$ e composto central rotacional. 


\section{MATERIAL E MÉTODOS}

\subsection{CURVA BINODAL DO SAB CONSTITUÍDO POR PEG/SULFATO DE SÓDIO}

A curva binodal foi construída pelo método da titulação, conforme descrito por Albertsson (1986), a partir de soluções estoque de PEG a $40 \%(\mathrm{~m} / \mathrm{m})$ e de sulfato de sódio a $20 \%(\mathrm{~m} / \mathrm{m})$. Adicionaram-se, aproximadamente, $1,5 \mathrm{~g}$ da solução de PEG a um tubo de ensaio mantido sobre balança analítica (GEHAKA, modelo AG-200) e, em seguida, a solução estoque de sulfato de sódio até o sistema ficar turvo. O aparecimento da turbidez indica que o sistema passou da região monofásica para a região bifásica. Conhecendo-se as massas adicionadas ao tubo de ensaio, calculam-se as concentrações de PEG e de sulfato de sódio necessárias para a mudança de fases. Obtém-se, com isso, o primeiro ponto da curva binodal. Os pontos adicionais foram obtidos pela adição alternada de água e sulfato de sódio, já que a água faz com que o sistema retorne à região monofásica. Isso foi feito até a concentração de PEG ficar próxima de zero. A partir desses valores de concentração de PEG e concentração de sal, obtém-se a denominada curva binodal.

\subsection{PREPARO DOS SISTEMAS AQUOSOS BIFÁSICOS}

Sistemas aquosos bifásicos foram obtidos pela mistura de solução aquosa de PEG 6.000 g.mol-1 ${ }^{-1}(40 \%$ em massa) e do sal sulfato de sódio ( $20 \%$ em massa). Os valores de pH das soluções estoques de PEG e sulfato de sódio foram ajustados com $\mathrm{NaOH}$ e $\mathrm{H}_{2} \mathrm{SO}_{4}$ e conferidos em ph-metro (QUIMIS, modelo Q-400A) até serem obtidos os valores desejados.

$\mathrm{Em}$ tubos de centrífuga graduados de $14 \mathrm{ml}$, foram adicionadas quantidades adequadas de PEG e sal de forma a se obter uma massa total fixa para o sistema de $10 \mathrm{~g}$. Após isso, a mistura foi agitada em vórtice por aproximadamente dois minutos e centrifugada a $2.000 \mathrm{~g}$ por 20 minutos. $\mathrm{Na}$ sequência, o sistema foi mantido em repouso por cerca de 24 horas em uma estufa BOD, na temperatura desejada, para alcançar o equilíbrio das fases. Em seguida, as fases resultantes foram coletadas por meio de uma seringa de $5 \mathrm{ml}$ (agulha de $10 \mathrm{~cm}$ ). Primeiro, retirou-se a fase superior, introduzindo a agulha da seringa vagarosamente, mantendo por 30 minutos e deixando uma camada de $0,5 \mathrm{~cm}$ acima da interface, para não perturbar o sistema. Após uma hora, a fase inferior foi coletada pela seringa deslizando suavemente pela parede do tubo até metade da fase, para evitar a perturbação da interface. As fases coletadas foram acondicionadas separadamente, para posterior montagem dos SABs, nos quais foram realizados os experimentos de partição. Esse procedimento foi realizado com o objetivo de manter a relação do volume de fases próximo a 1,0.

\subsection{PARTIÇÃO DA PROTEÍNA EM SAB}

Os experimentos de partição foram realizados em tubos de centrífuga de $14 \mathrm{ml}$, usando $2,5 \mathrm{ml}$ das fases superior e inferior pré-equilibradas dos SABs, de acordo com cada condição experimental definida.

Adicionou-se aos tubos um volume de $50 \mu \mathrm{l}$ de uma solução de BSA ( $50 \mathrm{mg} / \mathrm{ml})$, os quais foram agitados em vórtice. Em seguida, os sistemas foram centrifugados a $4000 \mathrm{~g}$ por 15 minutos e os tubos foram mantidos em repouso em estufa BOD por três horas em temperatura controlada. Após a partição da proteína, as fases superior e inferior foram coletadas e realizou-se a quantificação da concentração da proteína particionada em cada fase.

\subsection{PLANEJAMENTO EXPERIMENTAL}

Para o desenvolvimento do experimento, ele foi dividido em duas etapas. Na primeira, avaliou-se a influência dos fatores $\mathrm{pH}$, temperatura, concentração de sal e concentração de PEG sobre a partição da BSA. Para tanto, aplicou-se um delineamento fatorial fracionado $\left(2^{4-1}\right)$ constituído 
de 28 experimentos com quatro repetições no ponto central para estimativa do erro experimental. Esse tipo de delineamento constitui-se de um número pequeno e predeterminado de experimentos, que combinam adequadamente os fatores em estudo (BIASOLI, 2005). Os fatores e níveis estudados no delineamento fatorial fracionado são apresentados na tabela 1.

TABELA 1 - FATORES E NÍVEIS DO DELINEAMENTO FATORIAL FRACIONADO (24-1)

\begin{tabular}{cccc}
\hline & & Nível & \\
\hline Fator & -1 & 0 & +1 \\
\hline $\mathbf{p H}$ & 7,0 & 8,0 & 9,0 \\
\hline $\mathbf{T}$ & 25 & 30 & 35 \\
\hline$\% \mathbf{C}$ & 8,0 & 9,5 & 12,0 \\
\hline$\%$ PEG & 10,0 & 12,0 & 14,0 \\
\hline
\end{tabular}

$\mathrm{T}=$ temperatura $\left({ }^{\circ} \mathrm{C}\right), \% \mathrm{C}=$ concentração de sal $(\% \mathrm{~m} / \mathrm{m}), \%$ PEG = concentração de PEG $(\% \mathrm{~m} / \mathrm{m})$.

Os dados obtidos no experimento fatorial fracionado foram submetidos à análise de variância (ANOVA), ao nível de significância de 5\%.

$\mathrm{Na}$ segunda etapa, empregou-se um delineamento composto central rotacional (DCCR) com novos valores para as variáveis a fim de otimizar o processo de extração. Apenas os fatores significativos $(p<0,05)$ no primeiro experimento foram avaliados.

Os resultados foram submetidos à análise de regressão e então se obteve um modelo matemático que prediz o valor do coeficiente de partição da proteína em função dos níveis dos fatores testados. O nível de significância do coeficiente de regressão foi obtido pelo teste de Student (t). Todas as análises estatísticas foram realizadas utilizando o pacote estatístico Statistical Analysis System (SAS) ${ }^{\circledR}$, versão 9.0, procedimento PROCGLM (SAS Institute Inc., Cary, NC, EUA).

\subsection{QUANTIFICAÇÃO DA PROTEÍNA}

A concentração da proteína particionada em cada fase foi determinada pelo método de BRADFORD (1976).

\subsection{COEFICIENTE DE PARTIÇÃO}

O coeficiente de partição (K) foi determinado pela equação (1):

$$
K=\frac{[X]_{\text {sup }}}{[X]_{\text {inf }}}
$$

Onde: $[X] \sup [X] \sup \mathrm{e}[X] \inf \amalg[X] \inf$ são concentrações de equilibrio da proteína particionada na fase superior e inferior, respectivamente.

\section{RESULTADOS E DISCUSSÃO}

\subsection{CURVA BINODAL DO SISTEMA PEG/SULFATO DE SÓDIO}

A curva binodal do sistema PEG/sulfato (figura 1) foi obtida visando orientar a definição das possíveis concentrações de PEG (\% PEG) e do sal sulfato de sódio (\% sulfato) a serem utilizadas nas diversas extrações, que possibilitassem a separação de fases. Como eram inúmeras as concentrações possíveis, utilizaram-se na extração as menores quantidades de PEG e sulfato de 
sódio, com a condição de que se encontrassem na região bifásica (acima da binodal) do diagrama de fases. O sulfato de sódio foi encontrado em maior concentração na fase mais densa de fundo, enquanto PEG foi encontrado em maior concentração na fase superior.

\subsection{AVALIAÇÃO DA PARTIÇÃO DA BSA}

Com o intuito de investigar quais fatores tiveram efeito significativo na partição da proteína BSA, foi feita uma análise de variância (ANOVA) para o fatorial fracionado $2^{4-1}$. Os resultados da ANOVA referentes ao coeficiente de partição da BSA estão apresentados na tabela 2.

\section{TABELA 2 - RESULTADO DA ANOVA PARA O COEFICIENTE DE PARTIÇÃO DA BSA EMPREGANDO O FATORIAL $2^{4-1}\left(C V=28,89 \%\right.$ E $\left.R^{2}=99,83 \%\right)$}

\begin{tabular}{cccc}
\hline FV & GL & QM & P $>$ F \\
\hline pH & 1 & 6,353 & 0,0217 \\
\hline T & 1 & 70,409 & 0,0007 \\
\hline C & 1 & 52,943 & 0,0010 \\
\hline PEG & 1 & 0,024 & 0,8021 \\
\hline Modelo & 3 & 21,445 & 0,5355 \\
Erro & 24 & 22,445 & 0,0017 \\
\hline Falta de ajuste & 21 & 29,081 & \\
\hline Erro puro & 3 & 0,327 & \\
\hline Total & 27 & & \\
\hline
\end{tabular}

$\mathbf{G L}=$ graus de liberdade; $\mathbf{Q M}=$ quadrado médio; $\mathbf{F}$ = teste de Student; $\mathbf{P}=$ probabilidade; $\mathbf{R}^{2}$ = coeficiente de determinação

Os valores de probabilidade apresentados na tabela 2 indicam que as variáveis $\mathrm{pH}$, temperatura e concentração do sulfato de sódio interferem significativamente $(p<0,05)$ na partição da BSA. Já a concentração de PEG não interfere significativamente $(p>0,05)$ na partição da BSA.

\subsection{OTIMIZAÇÃO DO COEFICIENTE DE PARTIÇÃO DA BSA}

Com base nos resultados do coeficiente de partição da primeira etapa, aplicou-se à segunda etapa um delineamento composto central rotacional (DCCR) para otimizar os valores das variáveis significativas, tomando-se os valores de $\mathrm{pH} 6,66$ a 8,34 , temperatura de $23,30^{\circ} \mathrm{C}$ a 31,70 ${ }^{\circ} \mathrm{C}$ e concentração de sal de $8,66 \%$ a 10,34\%.

Por apresentar efeito não significativo $(p>0,05)$ na partição da BSA, a concentração de PEG foi fixada no DCCR em $12 \%$, sendo um valor não elevado e que assegura a formação da região bifásica para uma ampla faixa de concentração de sal.

Na tabela 3 são apresentados as condições utilizadas no DCCR e os dados experimentais para os valores do coeficiente de partição da BSA obtidos em SAB PEG + sulfato de sódio, concentração de PEG de $12 \%$. Os valores do coeficiente de partição da BSA variaram de 0,068 a 3,486 , sendo observado que os maiores valores foram obtidos nos SABs de maior valor de temperatura. Nesses pontos, observa-se que a proteína concentra-se preferencialmente na fase superior, enquanto, para os menores valores de temperatura e, consequentemente, de $\mathrm{K}$, a proteína concentra-se na fase inferior. 
TABELA 3 - RESULTADOS EXPERIMENTAIS OBTIDOS PARA O COEFICIENTE DE PARTIÇÃO DA BSA EMPREGANDO UM DCCR

\begin{tabular}{|c|c|c|c|}
\hline $\mathrm{T}\left({ }^{\circ} \mathrm{C}\right)$ & $\mathrm{pH}$ & $\%$ C & $\mathrm{K}$ \\
\hline 23,3 & 7,50 & 9,50 & 1,060 \\
\hline 25,0 & 7,00 & 9,00 & 0,068 \\
\hline 25,0 & 7,00 & 10,0 & 0,194 \\
\hline 25,0 & 8,00 & 9,00 & 0,197 \\
\hline 25,0 & 8,00 & 10,0 & 0,441 \\
\hline 27,5 & 6,66 & 9,50 & 0,314 \\
\hline 27,5 & 7,50 & 8,66 & 1,575 \\
\hline 27,5 & 7,50 & 9,50 & 0,463 \\
\hline 27,5 & 7,50 & 9,50 & 0,398 \\
\hline 27,5 & 7,50 & 9,50 & 0,234 \\
\hline 27,5 & 7,50 & 9,50 & 0,478 \\
\hline 27,5 & 7,50 & 10,34 & 0,486 \\
\hline 27,5 & 8,34 & 9,50 & 0,353 \\
\hline 30,0 & 7,00 & 9,00 & 0,611 \\
\hline 30,0 & 7,00 & 10,00 & 1,935 \\
\hline 30,0 & 8,00 & 9,00 & 0,971 \\
\hline 30,0 & 8,00 & 10,00 & 3,486 \\
\hline 31,7 & 7,50 & 9,50 & 1,935 \\
\hline
\end{tabular}

Os resultados do DCCR obtidos experimentalmente foram submetidos a análise de variância e análise de regressão, a fim de se verificar a influência dos fatores estudados sobre a partição da proteína BSA. Os fatores temperatura e concentração de sal foram significativos $(p<$ $0,10)$, para análise de regressão.

A equação para a otimização do coeficiente de partição $(K)$ da proteína BSA é:

$$
K=168,68836-8,23719 T-12,90462 C+0,06981 T^{2}+0,4912 T C
$$

Onde: T é a temperatura $\left({ }^{\circ} \mathrm{C}\right)$ do sistema e $\mathrm{C}$ é a concentração de sal do sistema (\%).

A significância estatística da equação (2) foi verificada pelo teste $F$, e os resultados da análise de variância (ANOVA) encontram-se na tabela 4. 
TABELA 4 - ANOVA PARA O MODELO DE SUPERFÍCIE DE RESPOSTA

\begin{tabular}{cccccc}
\hline FV & GL & SQ & QM & F Cal & P $>$ F \\
\hline Modelo & 4 & 13,01468 & 3,25367 & 4,01 & 0,0248 \\
\hline Falta de ajuste & 10 & 9,89493 & 0,98949 & 4,49 & 0,1217 \\
\hline Erro & 13 & 10,55678 & 0,81206 & & \\
\hline Total & 17 & 23,57146 & & &
\end{tabular}

$\mathbf{G L}$ = graus de liberdade; $\mathbf{S Q}=$ soma dos quadrados; $\mathbf{Q M}$ = quadrado médio; $\mathbf{F}=$ teste de Student; $\mathbf{P}$ = probabilidade

A falta de ajuste foi não significativa, ou seja, o modelo não apresentou falta de ajustamento aos dados experimentais a um nível de significância de 5\%. A partir da análise de regressão realizada (tabela 5), para os dados experimentais do DCCR, pode ser observado que, de acordo com o teste $t$ para a significância dos parâmetros, o fator temperatura teve um efeito linear e quadrático significativo $(p<0,10)$ na partição da proteína BSA, enquanto a concentração de sal foi significativa $(p<0,10)$ no efeito linear. A interação dos fatores temperatura e concentração de sal também foi significativa a um nível de $10 \%$ de probabilidade. O valor do desvio médio absoluto e do erro máximo entre os dados estimados pelo modelo e os dados experimentais foi de 0,496 e 1,117, respectivamente.

\section{TABELA 5 - SIGNIFICÂNCIA DOS COEFICIENTES DE REGRESSÃO PARA O COEFICIENTE DE PARTIÇÃO (K)}

\begin{tabular}{ccccc}
\hline Termo do modelo & Estimativa & Erro-padrão & Valor $\mathbf{P}$ & $\mathbf{P}|\mathbf{t}|$ \\
\hline Intercepto & 168,68836 & 72,98432 & 2,31 & 0,0379 \\
\hline $\mathrm{T}$ & $-8,23719$ & 3,24044 & $-2,54$ & 0,0246 \\
\hline $\mathrm{C}$ & $-12,90462$ & 7,02622 & $-1,84$ & 0,0892 \\
\hline $\mathrm{T}^{2}$ & 0,06981 & 0,03911 & 1,78 & 0,0976 \\
\hline $\mathrm{CT}$ & 0,49120 & 0,25488 & 1,93 & 0,0761 \\
\hline
\end{tabular}

$\mathrm{T}=$ temperatura $\left({ }^{\circ} \mathrm{C}\right), \mathrm{C}=$ concentração de sal $(\% \mathrm{~m} / \mathrm{m})$

Os coeficientes negativos para $\mathrm{T}$ e $\mathrm{C}$ mostraram um efeito linear de diminuição da resposta $\mathrm{K}$, e o coeficiente $\mathrm{T}$ mostrou um efeito quadrático para aumentar a resposta $\mathrm{K}$, assim como a interação dos fatores.

A utilização da metodologia de superfície de resposta permite verificar o efeito das variáveis concentração de sal e temperatura do sistema em relação ao coeficiente de partição da proteína BSA. A figura 2 representa o comportamento do coeficiente de partição da proteína BSA em função da temperatura e concentração $\mathrm{Na}_{2} \mathrm{SO}_{4}$

A superfície de resposta mostra que um aumento na concentração de sulfato de sódio causou uma diminuição do coeficiente de partição em menor valor de temperatura. Entretanto, à medida que se aumentou a temperatura, foi observada uma elevação do coeficiente de partição da proteína.

Avaliando a presença da proteína no sistema, segundo CHEFTEL et al. (1989) e DUARTE et al. (1998), a natureza e a concentração de íons exercem efeitos significativos sobre a absorção de água e a solubilidade da proteína. Assim, pequenas concentrações salinas podem 
elevar a solubilidade da proteína devido ao aumento da solvatação. Por outro lado, quando os níveis de sal são elevados, predominam as interações água-sal, em detrimento das interações água-proteína, contribuindo para reduzir a solubilidade proteica. Essa redução das interações água-proteína e o aumento das interações água-sal favorecem as interações PEG-proteína, que dependem tanto da carga da proteína quanto da capacidade indutora do sal utilizado na formação das fases.

Alguns relatos têm descrito um aumento do coeficiente de partição com a temperatura (DIAMOND e HSU, 1992; FORCINITI et al., 1991). Outros autores afirmaram que o coeficiente de partição não apresentou dependência da temperatura (JOHANSSON et al., 1973; TJERNELD et al., 1985). A variação da temperatura do sistema promove mudanças na viscosidade das fases ou na estrutura dos polímeros, alterando a forma da curva binodal no diagrama de fases (CARVALHO, 2004) e influenciando o comportamento de partição das biomoléculas. Essa alteração na curva de equilíbrio pode proporcionar um aumento no comprimento das linhas de amarração, pois, com o aumento da temperatura, há transferência de água da fase superior para a fase inferior, o que resulta na diminuição da concentração de sal na fase inferior e no aumento da concentração de PEG na fase superior (ALBERTSSON, 1986), promovendo assim uma maior diferença entre as fases do sistema e, consequentemente, um valor de coeficiente de partição mais distante de 1,0. Com a elevação da temperatura, a densidade e a viscosidade dos SABs diminuem e, como consequência, aumenta a mobilidade da BSA na fase superior.

SILVA (2000) e ZAFARANI-MOATTAR et al. (2002) relatam que o aumento da temperatura provoca diminuição da solubilidade mútua. Em outras palavras, aumento da região bifásica no diagrama de equilíbrio. Também pode ser dito que o aumento da temperatura em alguns casos pode induzir à formação de duas fases, pois a separação das fases é um processo endotérmico (CARVALHO, 2004).

Pelo exposto, um aumento na temperatura significa a elevação do coeficiente de partição da proteína, pois há tendência à formação do SAB. As regiões onde foram obtidos valores mínimos de coeficiente de partição para a BSA correspondem a uma temperatura de $25^{\circ} \mathrm{C}$ e altas concentrações de sal ou condições extremas de temperatura $\left(30^{\circ} \mathrm{C}\right.$ a $\left.31,7^{\circ} \mathrm{C}\right)$ e médias concentrações de sal.

Dessa forma, pode-se concluir que a temperatura e a concentração de sal influenciaram na partição da proteína em questão.

\section{ABSTRACT \\ STUDY OF PARTITION OF BOVINE SERUM ALBUMIN IN AQUEOUS TWO-PHASE SYSTEMS}

In this work the partition of bovine serum albumin (BSA) was evaluated in two-phase aqueous systems (ATPS), composed of polyethylene glycol (PEG), sodium sulfate and water. Phase equilibrium data for aqueous two-phase systems PEG / sodium sulfate at room temperature were determined. It was also evaluated the influence of $\mathrm{pH}$, temperature, concentration of PEG and salt concentration on the partition of BSA, applied, initially, in a fractional factorial design. The results were submitted to analysis of variance, being verified the influence of variables $\mathrm{pH}$, temperature, and concentration $\mathrm{Na} 2 \mathrm{SO} 4$ on the BSA partition. Then a central composite rotational design (DCCR) was employed. The results obtained in DCCR were submitted to regression analysis and response surface, and the best conditions to optimize the partition of the protein were determined. The extraction of BSA by SAB using PEG/Sodium sulfate was effective, using reagents and process steps quite simple.

KEY-WORDS: PROTEIN, POLYMER, PARTITION, SALT. 


\section{CONCLUSÃO}

Dados do equilíbrio para sistemas aquosos bifásicos formados por PEG 6.000 e sulfato de sódio foram determinados para estabelecer as concentrações mínimas de PEG e sal, formadoras de fases. A extração da BSA pelo SAB utilizando PEG/sulfato de sódio mostrou-se eficaz, utilizando-se de reagentes e etapas de processos bastante simples. Foi possível determinar regiões dos fatores temperatura e concentração de $\mathrm{Na}_{2} \mathrm{SO}_{4}$ que maximizem o coeficiente de partição da BSA.

\section{REFERÊNCIAS}

1 ALBERTSSON, P. A. Partition of cell and macromolécules. New York: John Wiley, 1986.

2 AZEVEDO, A.M.; ROSA, P.A.J.; FERREIRA, I.F.; AIRES-BARROS, M.R. Chromatography-free recovery of biopharmaceuticals through aqueous two-phase processing. Trends in Biotechnology, v.27, n.4, p.240-247, 2007.

3 BIASOLI, P. K. Modelagem conjunta de média e variância em experimentos fracionados sem repetições utilizando GLM. 2005. 123 f. Dissertação (Mestrado em Engenharia de Produção). Universidade Federal do Rio Grande do Sul, 2005.

4 BRADFORD, M. M.; A rapid and sensitive method for the quantitation of microgram quantities of protein utilizing the principle of protein-dye binding. Analytical Biochemistry, n.72, n.1-2, p.248-254.

5 CARVALHO, C. Estudo de Equilíbrio de Fases para Sistemas Aquosos Bifásicos Compostos por Polietileno Glicol, Sais e Água. 2004. 70f. Dissertação (Mestrado em ciências e Tecnologia de Alimentos) - Departamento de Tecnologia de Alimentos, Universidade Federal de Viçosa, 2004.

6 CAVALCANTI, M. T.H.; PORTO, T. S.; NETO, B. B.; LIMA-FILHO, J. L.; PORTO, A. L. F.; PESSOA JR, A. Purification of a-toxin from Clostridium perfringens type $A$ in PEG-phosphate aqueous two-phase systems: a factorial study Journal Chemical Technology Biotechnology v. 83:158-162; 2008.

7 CHEFTEL, J. C., CUA, J. L., LORIENT, D., Proteínas alimentarias. Zaragosa: Editorial Acribia, p. 345, 1989.

8 DA SILVA, L. H. M.; DA SILVA, M. C. H. OU DA SILVA, M. D. H.; AMIM JUNIOR, J.; MARTINS, J. P.; COIMBRA, J. S. R.; MINIM, L. A. Hydrophobic effect on the partitioning of $[\mathrm{Fe}(\mathrm{CN}) 5(\mathrm{NO})] 2-$ and $[\mathrm{Fe}(\mathrm{CN}) 6] 3-$ anions in aqueous two-phase systems formed by triblock copolymers and phosphate salts. Separation and Purification Technology, v.60, n.1, p. 103112,2008

9 DIAMOND, A. D.; HSU, J. T. Aqueous Two Phase Systems for Biomolecule Separation. Advances in Biochemical Engineering, v.47, p.89-135, 1992

10 DUARTE, J. A.; CARREIRA, L. R.; JUNQUEIRA, G.R.; COELHO, J. V.; SILVESTRE, C. P. M.; Propriedades emulsionantes e solubilidade da caseína bovina: Efeito da adição de $\mathrm{NaCl}$. Ciências e tecnologia de alimentos, v.18, n.3, 1998.

11 FORCINITI, D.; HALL, C. K.; KULA, M. R. Dependência de temperatura do coeficiente de partição de proteínas em sistemas aquosos de duas fases. Bioseparation, v.2, n.2, p.115-128, 1991.

12 HATTI-KAUL, R. Aqueous Two-phase Systems: A General Overview. Molecular Biotechnology, v.19, n.3, p.269-277, 2001.

13 HUSTED, H.; KRONER, K. H.; KULA, M-R., Applications of Phase Partitioning in Biotechnology, in: Partitioning in Aqueous Two-Phase Systems. Theory, Methods, Uses and Applications to Biotechnology, p.529-587, 1985.

14 JOHANSSON, G.; HARTMAN, R.; ALBERTSSON, P. A. Partição de proteínas em duas fases sistemas contendo cobrado poly (ethylene glycol). European Journal of Biochemistry, v.33, n.2, p.379-386, 1973.

$15 \mathrm{KIM}, \mathrm{C}$. W.; RHA, C. Y. Interfacial tension of polyethylene glycol/potassium phosphate aqueous two-phase systems. Physics and Chemistry Liquids, v.38, n.1, p.25-43, 2000.

16 PESSOA-JR, A., KILIKIAN, B. V. Purificação de Produtos Biotecnológicos. Editora Manole Ltda, São Paulo, 2005.

17 SILVA, L. H.; MEIRELLES, A. J. A. Bovine serum albumin, $\alpha$-lactoalbumin and $\beta$-lactoglobulin partitionig in poly (ethyleno glycol) maltodetrin aqueous two-phase sytems. Carbohydrate Polymers, v.42, n.3, p.279-282, 2000.

18 SAS - SAS/STAT. Statistical Analysis System. User’s guide, version 9.0 (Compact Disc). Cary, 2004. 
19 TJERnElD, F., PERSSON, I.; ALBERTSSON, P. A. HAHN-HAGERDAL, B. A hidrólise enzimática de celulose em sistemas aquosos de duas fases. I. Partição de celulases de Trichoderma reesei. Biotechnology Bioengineering, v.27,n.7, p.1036-1043, 1985 .

20 ZAFARANI-MOATTAR, M. T.; GASEMI, J.; Liquid-liquid phase equilibria of aqueous two-phase systems containing polyethylene glycol and ammonium dihydrogen phosphate or diammonium hydrogen phosphate: experiment and correlation. Fluid Phase Equilibria, v.198, n.2, 281-291; 2002. 